\title{
CYCLE ÉVOLUTIF DE LERNANTHROPUS KROYERI VAN BENEDEN, 1851, Copépode branchial hématophage du Loup Dicentrarchus labrax (Linné, 1758) dans des populations naturelles et en élevage
}

\author{
P. CABRAL, F. COSTE et A. RAIBAUT*
}

RÉSUMÉ. Le cycle évolutif de Lernanthropus kroyeri Van Beneden, 1851, Copépode (Siphonostomatoida) parasite de Dicentrarchus labrax est entièrement décrit. Le cycle comprend deux stades libres naupliens, un stade copépodite infestant, quatre stades copépodites fixés et un stade préadulte. Les stades fixés sont caractérisés par l'absence de filament frontal. C'est le premier cycle évolutif de Copépodes Siphonostomatoida parasites de Poissons, décrit sans Chalimus.

\section{The life cycle of Lernanthropus kroyeri Van Beneden, 1851, a hemato- phagous Copepod of the gills of the sea bass in wild hosts and experimen- tal infections.}

SUMMARY. The life cycle of Lernanthropus kroyeri Van Beneden, 1851, copepod (Siphonostomatoida) parasite of Dicentrarchus labrax is described. The cycle comprises two nauplii, one infective copepodid, four fixed copepodids and one preadult. All the attached stages are caracterised by the lack of the frontal filament. This is the first description of a life cycle lacking a chalimus for Siphonostomatoida Copepods parasitic on fish.

Le Loup Dicentrarchus labrax est un Poisson marin couramment élevé dans plusieurs régions méditerranéennes et au cours des différentes phases de son développement, des cas de mortalités dus à des agents infectieux ou à des parasites, ont été signalés (Lambert et Maillard, 1974 ; Barnabé, 1976 ; Oliver, 1977 ; Tesseyre, 1979 ; Paperna et al., 1979 ; Paperna, 1980 ; Bragoni et coll. [sous presse]). En ce qui concerne les Copépodes qui infestent ce Poisson, nous avons observé à plusieurs reprises des actions pathogènes provoquées par un Caligide buccal, Caligus minimus Otto, 1921,

- Laboratoire d'Ichthyologie et de Parasitologie Générale, Université des Sciences et Techniques du Languedoc, F 34060 Montpellier Cedex.

Accepté le 5 juillet I $983_{3}$. 
chez des Loups en grossissement (observations personnelles) de même que chez des géniteurs. En plus de ce dangereux parasite, le Loup héberge trois autres espèces de Copépodes, à savoir Colobomatus labracis Delamare Deboutteville et Nunes, 1952, Lernanthropus kroyeri Van Beneden, 1851 et Caligus dicentrarchi n. sp. (Cabral, sous presse). Si la première espèce ne semble pas causer de dommages aux canaux muqueux céphaliques qui constituent son microhabitat, les deux autres en revanche, en raison de leur localisation (respectivement sur les filaments branchiaux et le corps) et de leur hématophagie, représentent un danger pour leur hôte en cas de pullulation. D'où la nécessité de bien connaître la biologie de ces parasites en vue de déterminer éventuellement quelques mesures prophylactiques destinées à éviter leur prolifération.

C'est dans ce but qu'il nous a semblé particulièrement important d'étudier le cycle évolutif de ces parasites et de préciser les facteurs intervenant sur la stratégie de l'infestation.

Ce travail, qui concerne Lernanthropus kroyeri, représente la première phase de l'étude, c'est-à-dire l'identification des différentes étapes du développement larvaire à partir d'infestations expérimentales et de stades larvaires prélevés sur des Loups provenant de milieux naturels.

\section{Matériel et méthodes}

Stades larvaires prélevés sur des poissons provenant de milieux naturels et d'élevages

Dicentrarchus labrax est un poisson commun sur le littoral et dans les lagunes du Golfe du Lion ; il fait de plus l'objet d'élevages dans plusieurs stations piscicoles de notre région. L'examen de nombreux poissons, en particulier pendant le printemps et la saison chaude, nous a permis de récolter différents stades larvaires de Lernanthropus kroyeri sur les branchies. Cependant, les tous premiers stades fixés, dont la vie est éphémère, étaient très rares, d'où la nécessité de réaliser des infestations expérimentales.

\section{Stades larvaires obtenus par infestation expérimentale}

De nombreux essais ont montré la fragilité des premiers stades libres de Lernanthropus kroyeri. Les meilleurs résultats ont été obtenus dans les conditions suivantes: les sacs ovigères sont délicatement détachés des femelles fécondées, placés dans de petits cristallisoirs de 25 ou $50 \mathrm{ml}$ contenant de l'eau de mer filtrée à $35 \%$ et à une température de $20^{\circ} \mathrm{C}$.

Dans ces conditions, les premiers copépodites apparaîssent au bout de trois jours. Les infestations ont lieu le quatrième ou le cinquième jour dans des bacs de vingt litres, sur des poissons de 12 à 15 centimètres de longueur totale et toujours à une température constante de $20^{\circ} \mathrm{C}$. Les poissons ont été en majorité examinés durant les trois premiers jours après l'infestation afin de déterminer les premiers stades du développement du Copépode. 


\section{Description du cycle}

Le cycle comprend deux stades nauplii libres, un stade copépodite infestant, quatre stades copépodites fixés, un stade préadulte et un stade adulte. Tous les stades fixés sont accrochés aux filaments branchiaux. La différenciation sexuelle externe est observable dès le premier stade copépodite fixé, bien que les larves mâles et femelles soient à ce stade, très proches morphologiquement.

\section{Nauplius I}

Le corps est sphérique avec une paire de balanciers postérieurs (fig. 1). L'antennule trisegmentée, a une petite soie lisse sur les deux premiers segments et deux longues soies pennées apicales sur le segment terminal ( $f i g .2$ ).

L'antenne est biramée. L'endopodite est bisegmenté, le segment apical porte deux longues soies armées sur leur face externe, une soie rudimentaire apicale et une petite soie lisse médiane. L'exopodite est composé de quatre ou cinq segments inégaux et porte quatre longues soies pennées sur leur face externe ( fig. 3).

La mandibule est biramée. L'endopodite est bisegmenté avec deux longues soies apicales. L'exopodite, composé de quatre segments, porte quatre longues soies pennées (fig. 4).

Le nauplius I mesure entre 160 et $180 \mu \mathrm{m}$.

\section{Nauplius II}

Le corps est allongé, ovalaire (fig. 5). L'antennule est trisegmentée : trois soies courtes latérales et une soie courte apicale, toutes lisses, sont portées par le segment terminal en plus des deux longues soies apicales (fig. 6).

L'antenne et la mandibule sont proches de celles du nauplius I. Elles en diffèrent essentiellement par l'allongement de la soie lisse à l'apex de l'endopodite antennaire ( fig. 7) et par l'apparition d'une courte soie lisse sur le deuxième article de l'endopodite mandibulaire ( $f i g .8$ ).

La taille du nauplius II varie entre 240 et $320 \mu \mathrm{m}$.

\section{Copépodite infestant}

Le corps est allongé avec un céphalothorax ovalaire (fig. 65). La partie postérieure est constituée de quatre segments. Le premier, qui porte la deuxième paire de pattes, est large. Le deuxième contient les rudiments de la troisième paire de pattes. Les deux segments suivants sont aussi longs que larges, le dernier portant les uropodes (fig. 52).

L'antennule, robuste, est composée de sept segments assez indistincts avec une flexion au niveau du segment basal. Dix-sept soies lisses et longues pour la plupart d'entre elles, sont réparties tout le long de l'appendice avec onze soies sur les deux derniers segments (fig. 9). 

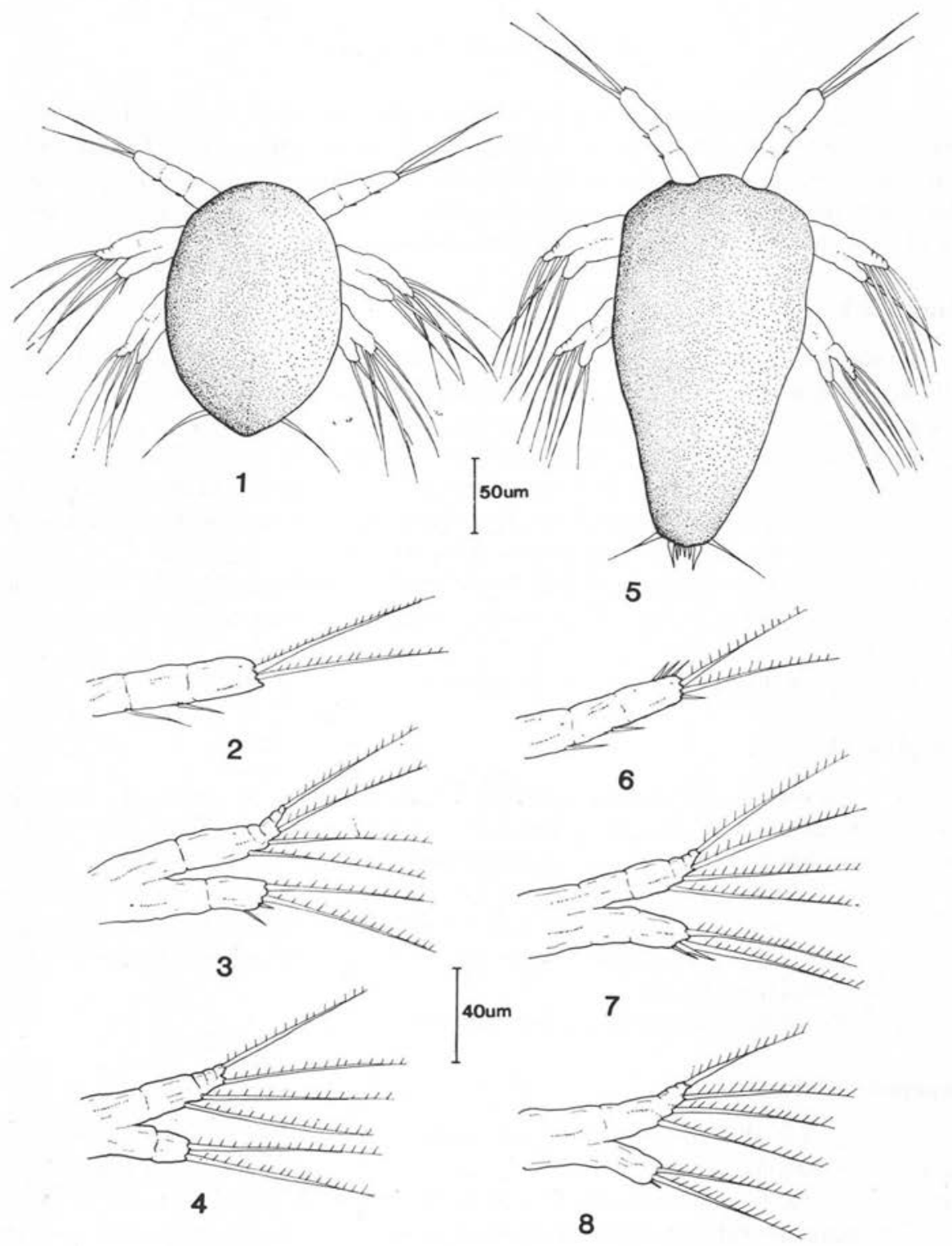

PLANChE I. - Stades naupliens I et II.

Fig. I à 4 : Nauplius I, face dorsale. -1 : habitus ; $2:$ Antennule ; $3:$ Antenne ; $4:$ mandibule.

FIg. 5 à 8 : Nauplius II, face dorsale. -5 : habitus; $6:$ Antennule ; $7:$ Antenne ; $8:$ mandibule. 

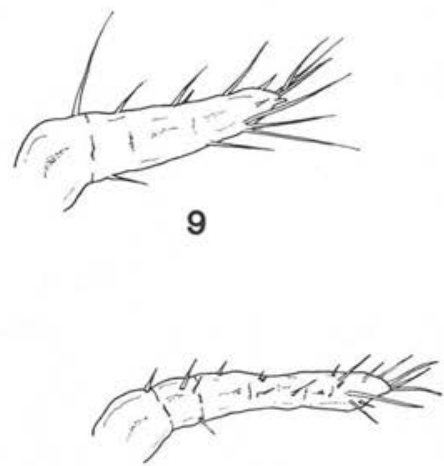

11

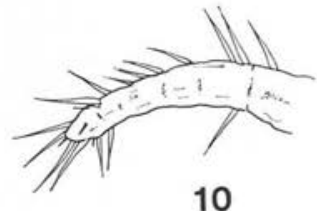

10
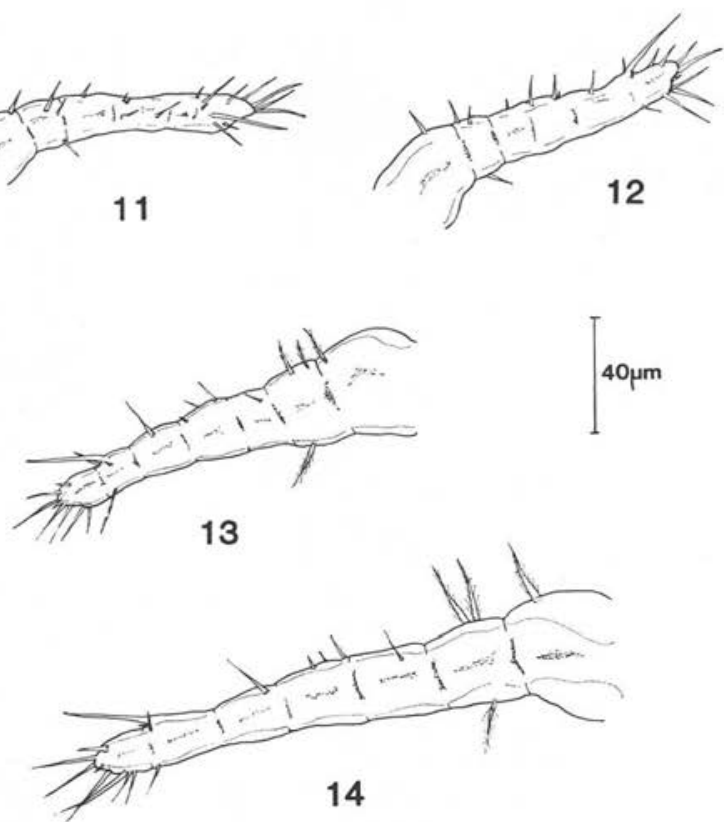

Planche II. - Antennules, face ventrale

Fig. 9. Copépodite infestant. - Fig. Io. I $^{\mathrm{er}}$ copépodite fixé, droite. - Fig. II. $2^{\mathrm{e}}$ copépodite fixé, gauche. - Fig. I2. $3^{\text {e }}$ copépodite fixé, gauche. - Fig. 13. $4^{\mathrm{e}}$ copépodite fixé, droite. Fig. I4. Adulte juvénile, droite.

L'antenne est relativement importante par rapport au corps. Sur la face ventrale de la partie proximale s'insère une courte soie épaisse. Le crochet est très fin et porte deux épines robustes, respectivement ventrales et médianes (fig. 15).

La mandibule est composée de huit dents identiques peu discernables ( $f i g .61$ ).

La maxillule est biramée. L'exopodite, plus petit que l'endopodite, porte deux soies lisses. Sur l'endopodite s'insèrent deux soies courtes latérales et une soie longue médiane, toutes lisses (fig. 58).

La maxille est composée de trois parties distinctes. Une base robuste, une partie médiane élancée avec une soie rudimentaire dorsale et une partie terminale en forme de spatule avec deux rangées de sétules dorsales ( $f i g$. 23). 


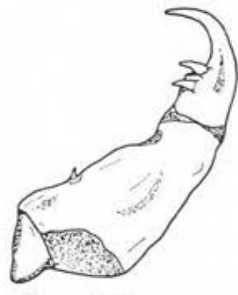

15
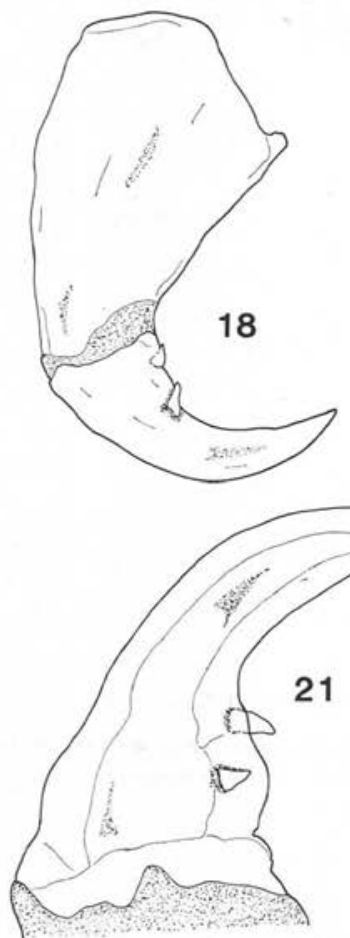
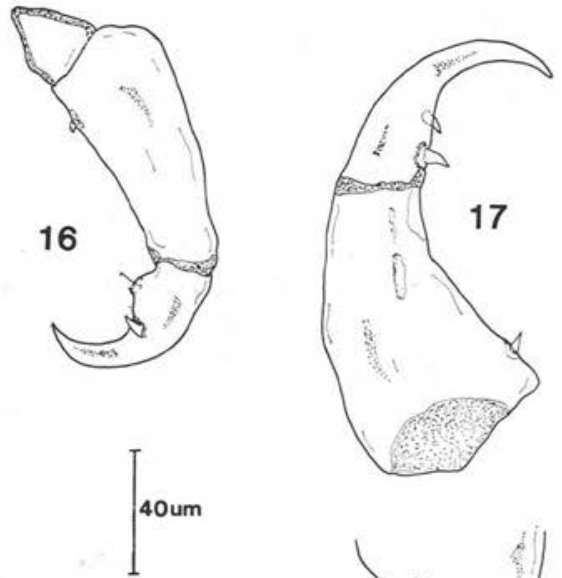

40um

19
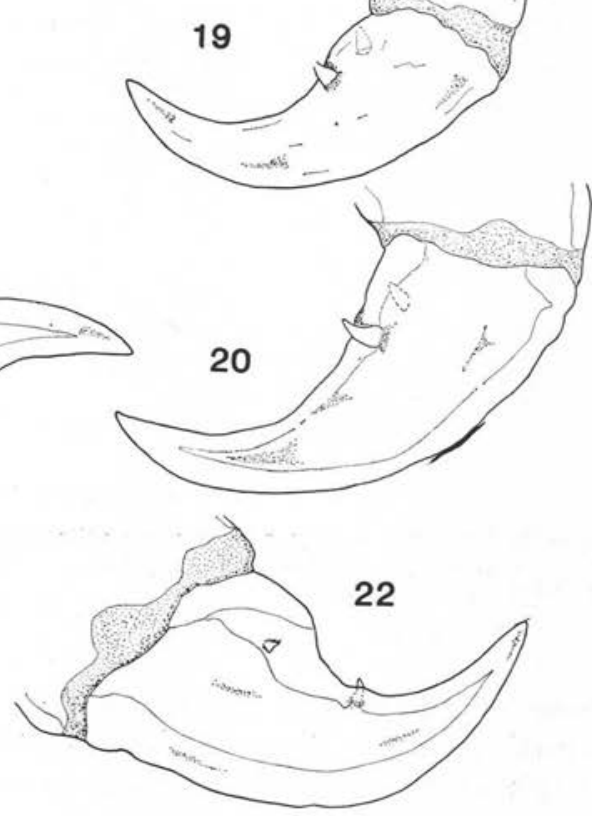

Planche III. - Antennes.

Fig. I5. Copépodite infestant, gauche, face ventrale. - Fig. I6. I er Copépodite fixé, gauche, face dorsale. - Fig. I7. $2^{\mathrm{e}}$ Copépodite fixé, droite, face ventrale. - Fig. I8. $3^{\mathrm{e}}$ Copépodite fixé, droite, face dorsale. - Fig. I9. $4^{\mathrm{e}}$ Copépodite fixé, gauche, face dorsale. - Fig. 20. Préadulte, gauche, face dorsale. - Fig. 21. Adulte juvénile mâle, droite, face ventrale. - Fig. 22. Adulte juvénile femelle, gauche, face ventrale. 
Le maxillipède est divisé en trois parties : une partie proximale allongée et robuste, une partie médiane renflée et un article distal conformé en un puissant crochet à extrémité bifurquée à la base duquel s'insère une courte soie lisse (fig. 30).

La première patte est biramée. Le segment sympodial n'est pas armé. L'endopodite, unisegmenté, présente six longues soies plumeuses. L'exopode, également unisegmenté, porte sur le bord externe trois épines lisses suivies d'une soie plumeuse courte et de quatre longues soies plumeuses ( fig. 37).

La deuxième patte est biramée. Le segment sympodial et l'endopodite sont identiques à ceux de la première patte. L'exopode unisegmenté porte une petite épine latéro-externe, une courte soie lisse et quatre longues soies plumeuses (fig. 44).

La troisième patte est rudimentaire, réduite à une proéminence porteuse d'une soie plumeuse longue, externe, et d'une soie lisse, courte et externe ( fig. 52).

L'uropode porte cinq soies lisses (fig. 52).

Ces copépodites mesurent entre 320 et $380 \mu \mathrm{m}$ de longueur. Ils nagent activement et, mis en présence de Loups, ils se fixent au niveau des filaments branchiaux à l'aide de leurs antennes unciformes. Ce stade évolue ensuite progressivement vers la forme adulte en passant par cinq stades copépodites fixés. Alors que les copépodites libres ont strictement la même morphologie, dès la première mue sur les branchies des poissons nous avons pu déceler une différence dans la structure de la troisième patte thoracique nous permettant de distinguer le sexe de la larve.

\section{Premier stade copépodite fixé}

\section{Femelle}

Le céphalothorax conserve une taille importante tandis que la partie postérieure s'élargit et perd la segmentation observée chez le copépodite libre (fig. 66).

L'antennule ( fig. 10) conserve les sept segments du stade précédent. La chétotaxie varie quelque peu toutefois avec vingt et une soies réparties de la manière suivante : une soie sur le premier segment basal, trois soies sur le deuxième segment, une soie sur le troisième segment, trois soies sur le quatrième, une soie sur le cinquième, quatre soies sur le sixième et huit soies sur le septième segment.

L'antenne a une structure proche de celle du copépodite libre (fig. 16).

La mandibule (fig. 62) montre désormais à son extrémité des dents nettement discernables, au nombre de huit.

La maxillule (fig. 59) est caractérisée par la perte d'une soie au niveau de l'exopodite.

La maxille (fig. 24) s'épaissit. L'extrémité du dernier article devient pointue.

Le maxillipède ( $f$ ig. 31 ) devient plus puissant avec notamment un segment basal bien développé. Deux petites soies s'insèrent sur le deuxième article, en position ventrale et à la base du crochet.

La première et la deuxième patte thoracique ( fig. 38 et 45) subissent, à l'inverse des appendices précédents, des modifications importantes imputables à la vie fixée. Les deux pattes sont biramées, chaque rame étant uniarticulée. Les soies subissent des régressions dans la taille et dans le nombre. L'endopodite ne porte plus qu'une 

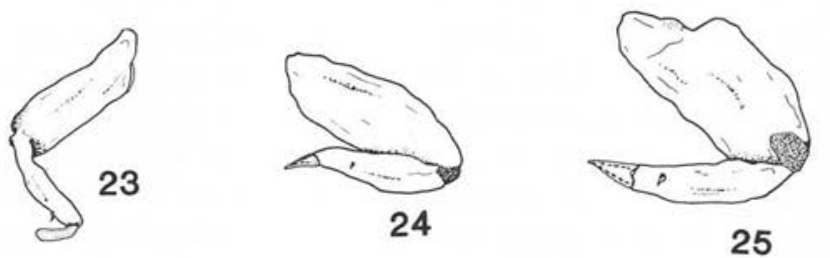

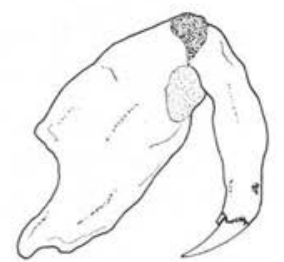

26

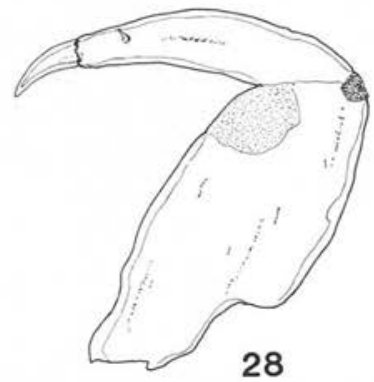

Planche IV. - Maxilles.

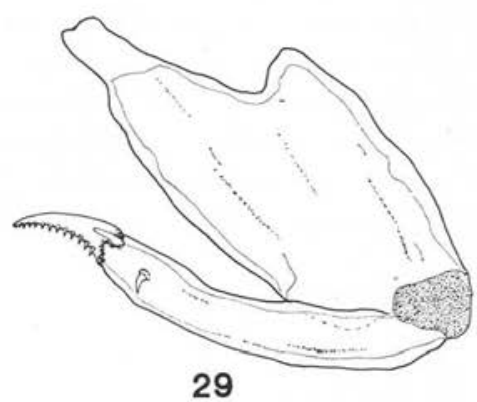

29

Fig. 23. Copépodite infestant, droite, face dorsale. - Fig. 24. I er Copépodite fixé, gauche, face dorsale. - Fig. 25. $2^{\mathrm{e}}$ Copépodite fixé, gauche, face dorsale. - Fig. 26. $3^{\mathrm{e}}$ Copépodite fixé, droite, face ventrale. - Fig. 27. $4^{\mathrm{e}}$ Copépodite fixé, gauche, face dorsale. - Fig. 28. Préadulte, gauche, face ventrale. - Fig. 29. Adulte juvénile, gauche, face dorsale.

soie tandis que l'exopodite de la première patte et de la deuxième patte sont armés respectivement de 5 et 4 épines.

La troisième patte thoracique ( fig. 53), rudimentaire chez le copépodite libre, prend un aspect lamellaire. La structure de cet appendice dont le bord interne est régulier, nous permet de différencier une larve femelle.

La quatrième patte thoracique ( fig. 53), inexistante chez le copépodite libre, apparaît sous la forme d'une expansion lobée, unique, latérale.

Le premier copépodite fixé femelle mesure entre 360 et $410 \mu \mathrm{m}$.

\section{Mâle}

La seule différence avec le premier stade copépodite fixé femelle réside dans la structure de la troisième patte thoracique dont le bord interne montre une légère dépression constante ( ig. 54).

Le premier copépodite mâle fixé a la même taille que le copépodite femelle. 

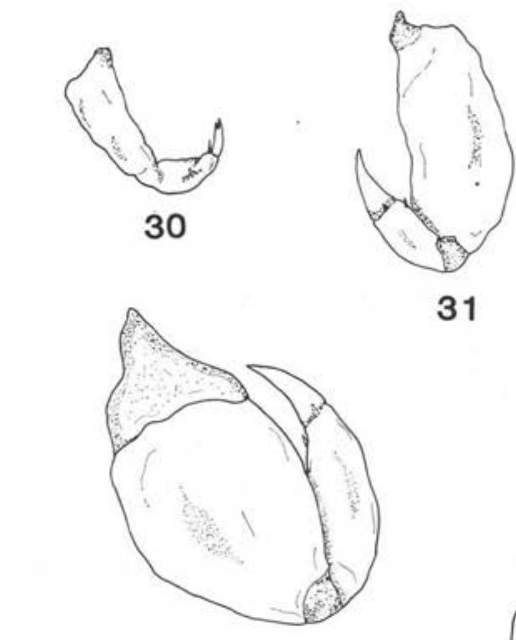

31

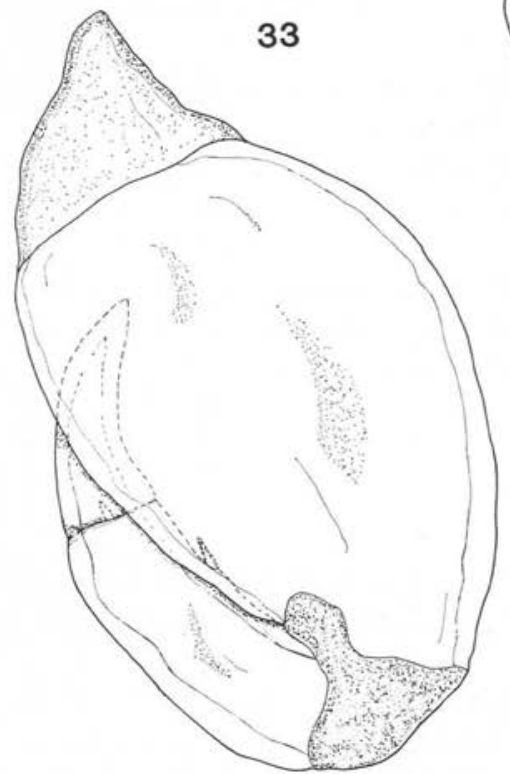

36
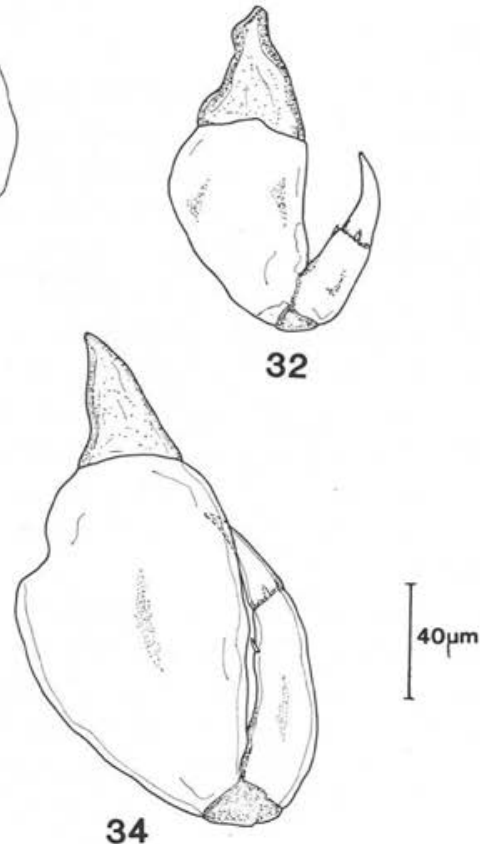

34

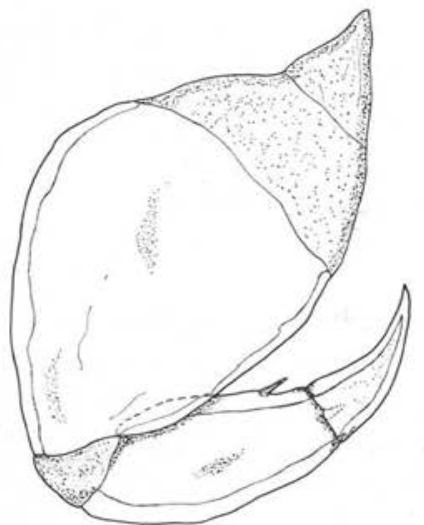

35

Planche V. - Maxillipèdes, face dorsale

Fig. 3o. Copépodite infestant, droite. - Fig. 31. I er Copépodite fixé, gauche. - Fig. 32. 2e Copépodite fixé, droite. - Fig. 33. $3^{\mathrm{e}}$ Copépodite fixé, droite. - Fig. $34 \cdot 4^{\mathrm{e}}$ Copépodite fixé, droite. - Fig. 35. Préadulte, droite. - Fig. 36. Adulte juvénile, gauche. 


\section{Deuxième stade copépodite fixé}

Femelle (fig. 67)

Le céphalothorax est toujours important par rapport à la longueur totale du corps. Celui-ci s'élargit au niveau des troisièmes pattes. La distinction mâle-femelle n'est plus délicate, la troisième patte du mâle étant totalement divisée à ce stade.

L'antennule ( fig. 11) est similaire à celle du stade précédent. La chétotaxie est identique mais les soies subissent une légère régression.

L'antenne ( fig. 17) conserve la même structure qu'au premier stade copépodite fixé. Seule la partie proximale s'élargit quelque peu.

La mandibule est identique ( fig. 63)

La maxillule acquiert sa structure définitive ( $f i g .60$ ) avec une soie courte sur l'exopodite, deux soies courtes et une soie longue sur l'endopodite.

La maxille est comparable à celle des stades précédents (fig. 25).

La maxillipède (fig. 32) s'élargit à sa partie basale mais conserve la même configuration.

La première et la deuxième patte thoracique sont strictement identiques à celles du premier stade copépodite fixé ( fig. 39 et 46).

La troisième patte thoracique s'allonge et prend un aspect lamellaire (fig. 55), tandis que la quatrième patte thoracique est bilobée (fig. 55).

Les uropodes conservent la même structure (fig. 55).

Mâle (fig. 73)

Les appendices sont identiques. Seule la troisième patte permet la distinction sexuelle, celle du mâle étant à ce stade nettement divisée ( fig. 56).

Femelles et mâles mesurent entre 410 et $480 \mu \mathrm{m}$.

\section{Troisième copépodite fixé}

Femelle

Le céphalothorax représente toujours environ la moitié de la longueur totale du corps. Les troisième et quatrième pattes s'allongent et s'élargissent nettement (fig. 68).

L'antennule (fig. 12) est semblable à celles des stades précédents. Le segment basal s'élargit et se renforce légèrement. La chétotaxie n'est pas modifiée.

L'antenne (fig. 18) conserve une structure identique. Le segment proximal s'élargit et perd la soie insérée à sa base. Les épines du crochet ont une position plus interne.

La mandibule et la maxillule sont identiques à celles du deuxième stade copépodite fixé.

La maxille ( $f i g$. 26) présente une courte soie supplémentaire à la base du crochet, mais conserve une structure semblable.

Le maxillipède (fig. 33) est similaire à ceux des précédents stades. 

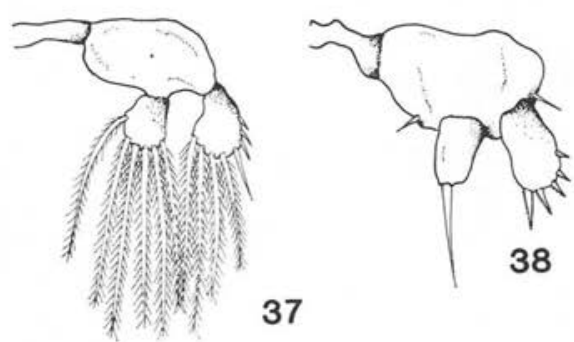

37
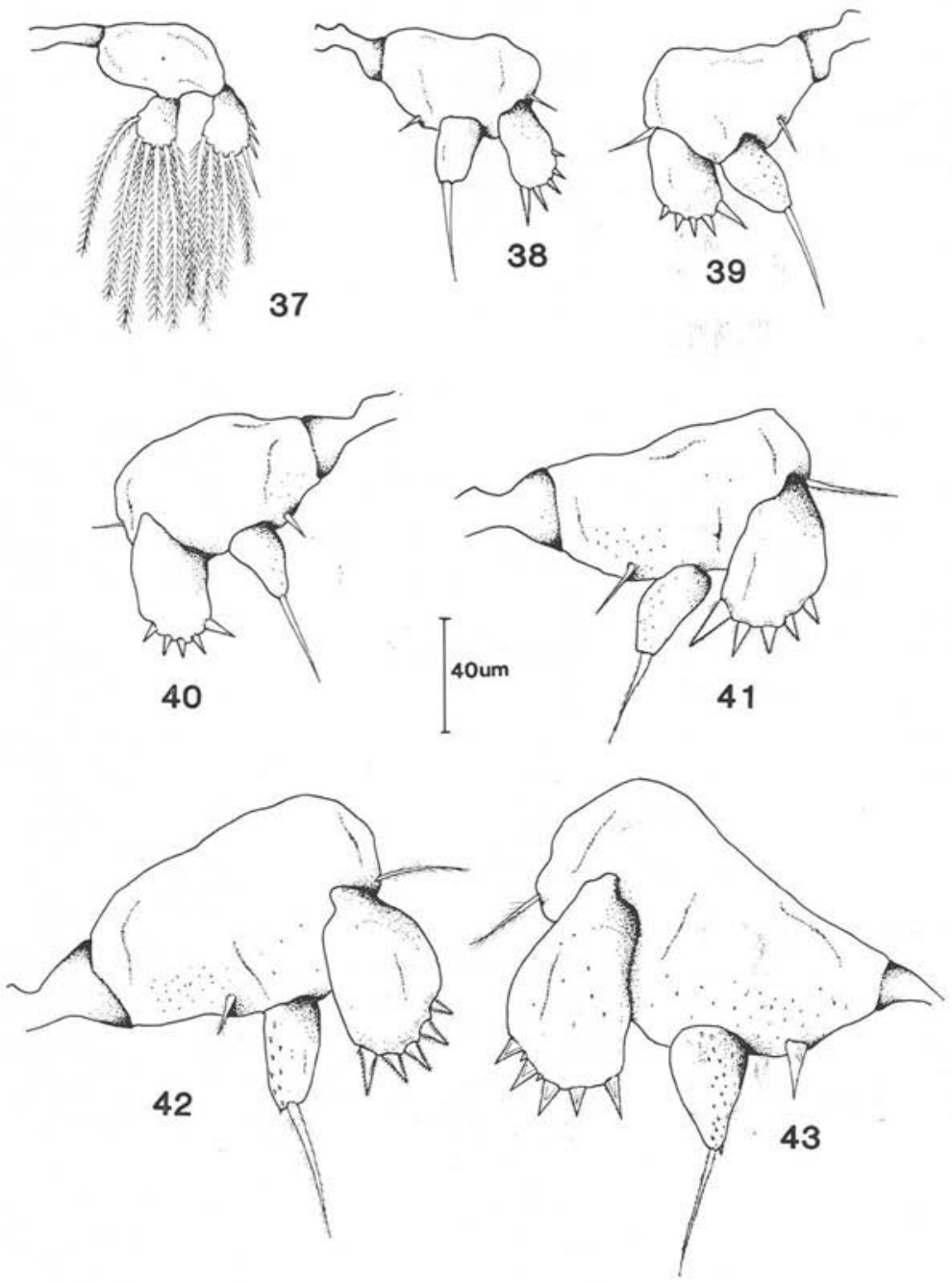

Planche VI. - Premières pattes thoraciques $\left(\mathrm{P}_{1}\right)$, face dorsale.

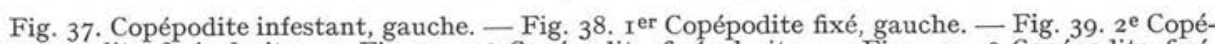
podite fixé, droite. - Fig. 40. $3^{\text {e }}$ Copépodite fixé, droite. - Fig. 4I. $4^{\mathrm{e}}$ Copépodite fixé, gauche. - Fig. 42. Préadulte, gauche. - Fig. 43. Adulte juvénile, droite.

La première et la deuxième patte thoracique ne subissent aucune modification importante (fig. 40 et 47).

La troisième patte thoracique ( $f$ ig. 68 ) s'allonge mais garde la même forme.

La quatrième patte n'atteint pas encore en longueur les uropodes (fig. 68).

Les uropodes conservent une structure identique. 

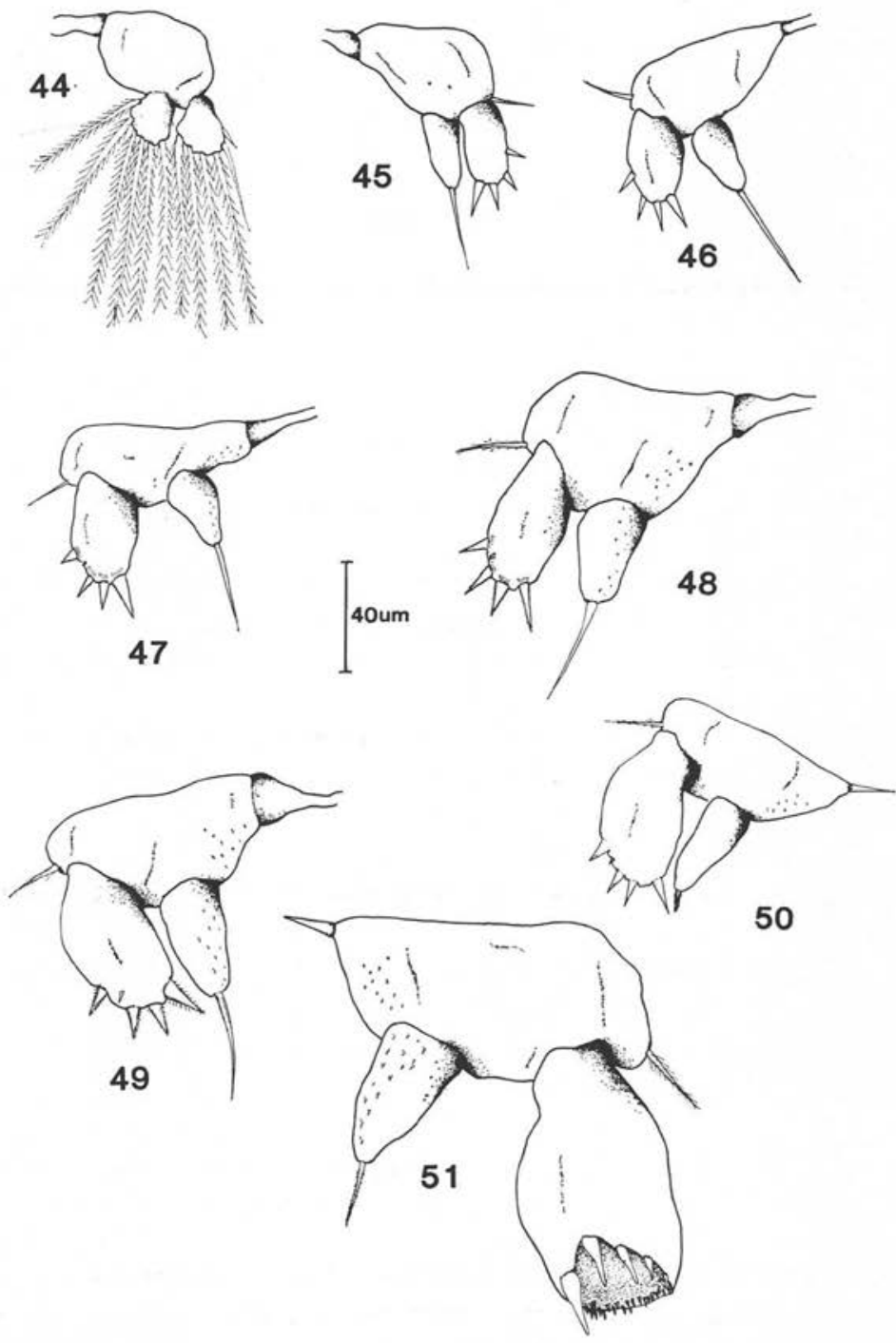

Planche VII. - Deuxièmes pattes thoraciques $\left(\mathrm{P}_{2}\right)$, face dorsale.

Fig. 44. Copépodite infestant, gauche. - Fig. 45. I $^{\mathrm{er}}$ Copépodite fixé, gauche. - Fig. 46. $2^{\mathrm{e}}$ Copépodite fixé, droite. - Fig. 47. $3^{\mathrm{e}}$ Copépodite fixé, droite. - Fig. $48.4^{\mathrm{e}}$ Copépodite fixé, droite. - Fig. 49. Préadulte, droite. - Fig. 50. Adulte juvénile femelle, droite. - Fig. 5 I. Adulte juvénile mâle, gauche. 
Mâle

Semblable à la femelle à l'exception de la troisième patte divisée (fig. 74).

Les femelles et les mâles mesurent entre 500 et $630 \mu \mathrm{m}$.

\section{Quatrième copépodite fixé}

Femelle

Le corps s'allonge mais le céphalothorax devient moins important ( $f i g .69)$. La partie postérieure s'élargit.

L'antennule ( $f$ ig. 13) ne subit aucune transformation.

L'antenne (fig. 19) est peu modifiée. Les épines du crochet sont relativement plus petites et en position interne.

La mandibule et la maxillule sont identiques à celles du stade copépodite 3 .

La maxille est semblable à celles des stades antérieurs ( fig. 27).

Le maxillipède est peu modifié ( fig. 34).

Les premières et deuxièmes pattes thoraciques ( $f i g .41$ et 48$)$ sont similaires à celles des précédents stades mais présentent des denticulations plus importantes. Une petite épine interne, peu visible jusque-là, apparaît entre les deux épines externes de l'exopodite de la deuxième patte.

Les troisièmes et quatrièmes pattes thoraciques (fig. 69) continuent leur croissance. La dernière paire arrive maintenant à hauteur des uropodes.

\section{Mâle}

La seule différence apparente se situe toujours au niveau de la troisième patte thoracique, bifide chez le mâle (fig. 75).

Les individus des deux sexes mesurent entre 650 et $850 \mu \mathrm{m}$.

\section{Préadulte}

Femelle

Ce stade, qui est en fait le cinquième copépodite fixé, est caractérisé par l'apparition des fentes génitales. Les gonades ne sont pas encore fonctionnelles. Le processus de céphalisation s'est intensifié et le segment génito-abdominal s'élargit ( fig. 70).

Les appendices sont très semblables à ceux des précédents stades (fig. 14, 20, $28,35,42$ et 49). La cinquième patte thoracique apparait, représentée par une soie dorsale au niveau du segment génital.

\section{Mâle}

Les fentes génitales apparaissent aussi à ce stade chez le mâle mais moins développées (fig. 76). Les appendices sont identiques à ceux de la femelle à l'exception de la troisième patte thoracique et de la cinquième patte qui n'apparaît pas.

Les préadultes mesurent entre 820 et $1200 \mu \mathrm{m}$ pour les mâles et 850-1 $390 \mu \mathrm{m}$ pour les femelles. 


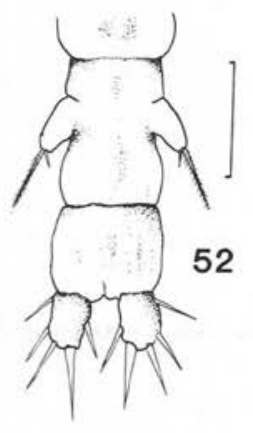

53

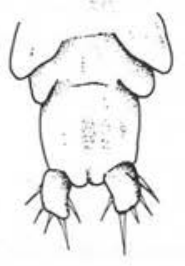

55

57
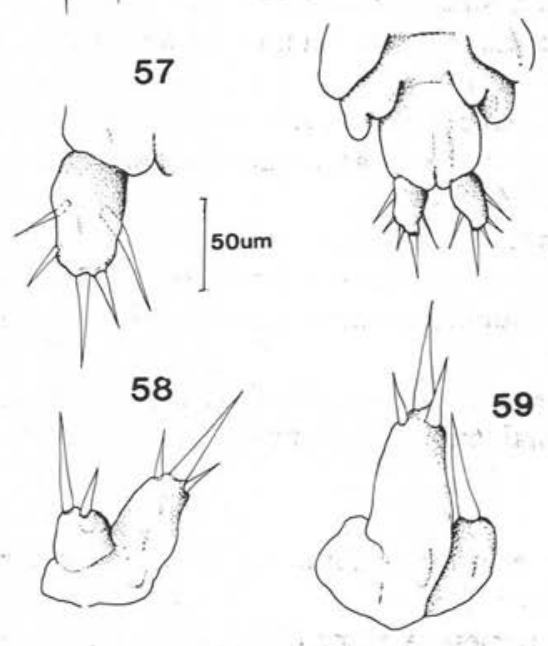

54

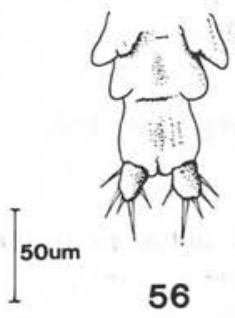

56
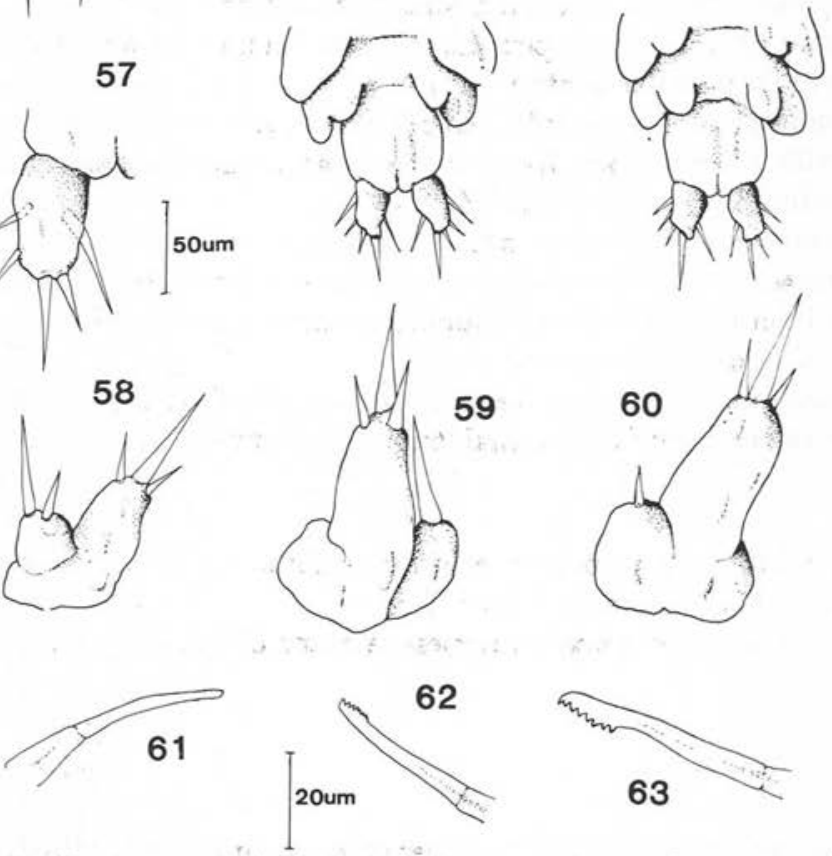

64

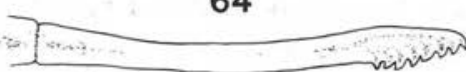

PLANCHE VIII.

FIG. 52 à 56 : Troisièmes et quatrièmes pattes thoraciques et segment génito-abdominal, face

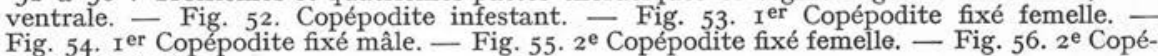
podite fixé mâle.

Fig. 57. Rame furcale du Préadulte.

FIG. 58 à 60 : Maxillules, face ventrale. - Fig. 58 . Copépodite infestant, droite. - Fig. 59. $\mathrm{I}^{\mathrm{er}}$

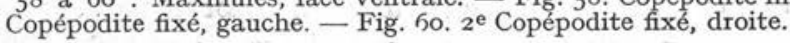

Fig. 6r à 64 : Mandibules. - Fig. 6r. Copépodite infestant. — Fig. 62. rer Copépodite fixé. Fig. 63 . $3^{\mathrm{e}}$ Copépodite fixé. - Fig. 64. Adulte juvénile. 
Adultes : formes juvéniles

Femelle (fig. 71)

Le processus de céphalisation est terminé. Le segment génital s'élargit et le bouclier dorsal se développe. Bien que les appendices soient identiques à ceux des formes adultes décrites par de nombreux auteurs (Scott et al., 1913, Kabata, 1979) et observés par nous mêmes, les formes juvéniles des femelles n'ont pas leur conformation définitive : les lobes du céphalothorax ne se sont pas encore développés, les troisièmes pattes thoraciques ne sont pas totalement recourbées et le bouclier dorsal n'est pas totalement formé. Ce n'est que lorsque les individus, qui jusqu'alors étaient accrochés sur la face externe des filaments branchiaux, se fixeront sur la face interne de ces mêmes filaments, que les lobes latéraux et les troisièmes pattes prendront leurs aspects caractéristiques.

L'antennule ( fig. 14) est identique au niveau de la chétotaxie. Seuls les renforcements cuticulaires apparaissent plus nettement.

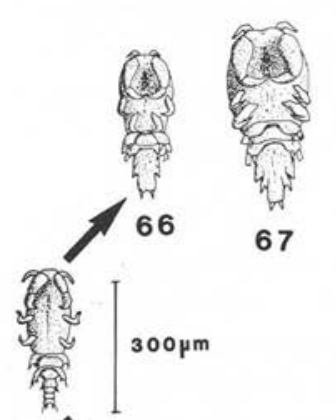

65

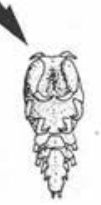

72

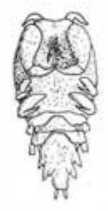

73

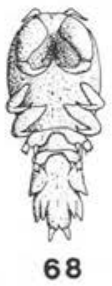

68

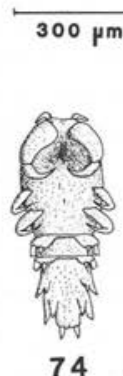

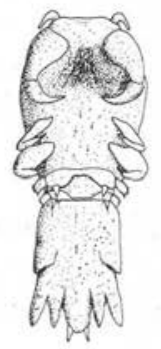

69

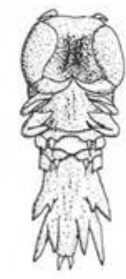

75
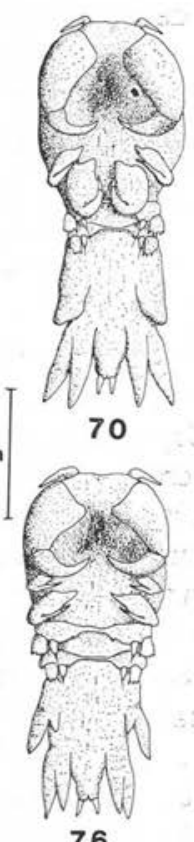

76
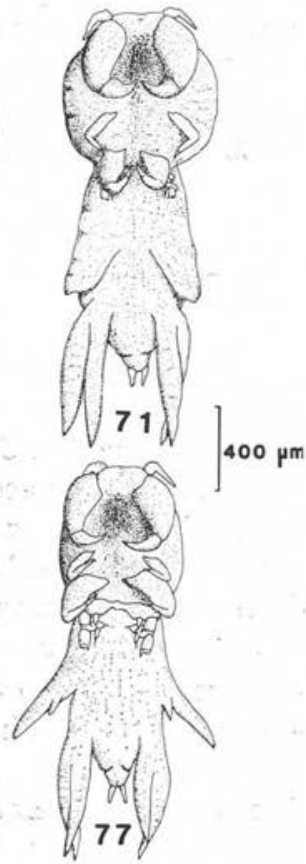

Planche IX. - Habitus, face ventrale.

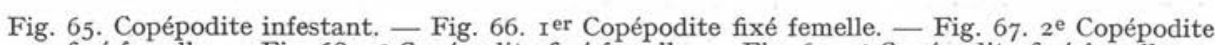
fixé femelle. - Fig. 68. $3^{\mathrm{e}}$ Copépodite fixé femelle. - Fig. $69.4^{\mathrm{e}}$ Copépodite fixé femelle. Fig. 70. Préadulte femelle. - Fig. 71. Adulte juvénile femelle. - Fig. 72. I er Copépodite fixé mẩle. - Fig. 73. $2^{\mathrm{e}}$ Copépodite fixé mâle. - Fig. $74 \cdot 3^{\mathrm{e}}$ Copépodite fixé mâle. - Fig. 75. $4^{\mathrm{e}}$ Copépodite fixé mâle. - Fig. 76. Préadulte mâle. - Fig. 77. Adulte juvénile mâle. 
L'antenne (fig. 22) est peu modifiée mais le crochet subit une nette réduction de sa taille relative. Les épines deviennent rudimentaires.

La mandibule et la maxillule ne sont pas modifiées.

La maxille ( fig. 29) est semblable à celles des autres stades mais les dents du crochet sont saillantes et robustes.

Le maxillipède ( fig. 36 ) conserve une structure similaire.

La première patte thoracique ( fig. 43) est peu modifiée.

La seconde patte thoracique (fig. 50) n'est plus unie par le segment interpodal. La soie distale de l'endopodite est réduite.

Les troisième et quatrième patte thoracique s'allongent et s'élargissent ( fig. 71).

La cinquième patte thoracique est formée d'une expansion dorsale prolongée par une longue soie.

Mâle

La conformation des juvéniles est, à l'inverse des femelles, très proche des formes adultes (fig. 77). Il n'y a pas de bouclier dorsal. Certains appendices présentent des différences par rapport à ceux des femelles. Il s'agit de l'antenne (fig. 21) avec un crochet plus long et de la deuxième patte thoracique (fig. 51) très transformée.

Le stade adulte, caractérisé par la disparition du segment interpodal de la deuxième patte thoracique, peut apparaître à partir de $1100 \mu \mathrm{m}$ chez les mâles et $1300 \mu \mathrm{m}$ chez les femelles.

\section{Discussion}

Le cycle évolutif de Lernanthropus kroyeri comprend une phase libre raccourcie, caractéristique des Copépodes parasites de Poissons et plus particulièrement des Siphonostomes, un Copépodite infestant armé d'antennes unciformes très développées lui permettant un accrochage efficace sur les filaments branchiaux et plusieurs stades copépodites fixés, très semblables morphologiquement au copépodite infestant, ne possédant pas de filament frontal, et accroché aux lamelles branchiales à l'aide de leurs antennes robustes.

Jusqu'à présent tous les cycles connus de Copépodes Siphonostomes parasites de Poissons, étaient caractérisés par la présence de stades larvaires fixés par un filament frontal, les Chalimus. C'est le cas chez les Caligidae (Wilson, 1905 ; Gurney, 1933 ; Lewis, 1963 ; Hwa, 1965 ; Izawa, 1969 ; Kabata, 1972 ; Boxshall, 1974...), les Lernaeopodidae (Kabata et Cousens, 1973 ; Kawatow et al., 1980...) et les Pennellidae (Grabda, 1963 ; Ho, 1966 ; Schram, 1979...). Cela a amené Kabata (1981) à considérer ce type de cycle comme caractéristique des Copépodes Siphonostomes parasites de Poissons. Ainsi, à propos du développement larvaire des Caligidae, il écrit : "The infective stage is the first of the next cycle segment, the Copepodid. The remainder of that segment (represented by Chalimus I-IV) passes in the attached condition, the chalimi being securely fixed to the host by means of the frontal filament, a structure 
unique to, and prevailing among Siphonostomatoida. (The report of some Siphonostomatoids developing without the benefit of this larval device require corroboration.) ”

Pourtant des Copépodites de Siphonostomes sont mentionnés sans filament frontal. C'est le cas de Dissonus nudiventris Kabata, 1965 et de Dichelesthium oblongum (Abilgaard, 1794) dont les copépodites infestants ont été décrits respectivement par Anderson et Rossiter (1969) et Kabata et al. (1977). D'où la remarque de Kabata (1981) : "This is very unusual for the Siphonostomatoids and could be seen as a primitive character $)$.

Dans l'état actuel de nos connaissances, la larve Chalimus a été rencontrée chez des Copépodes Siphonostomes appartenant à quatre familles, à savoir : les Lernaeoceridae, Les Pennellidae, les Caligidae et les Cecropidae (Grabda, 1973), ces deux dernières étant proches. Avec la découverte du cycle de Lernanthropus kroyeri, l'absence de filament frontal est relevée chez trois familles de Copépodes Siphonostomes, c'est-à-dire les Lernanthropidae, les Dichelesthiidae et les Dissonidae. Cela tend donc à montrer que les développements larvaires des Copépodes Siphonostomes sans Chalimus ne constituent pas une exception.

Il est toutefois certain que la présence d'une telle larve Chalimus ne se rencontre que chez des Copépodes Siphonostomes et, à cet égard, elle peut être considérée comme une étape évolutive supplémentaire. En fait, on peut remarquer que le filament frontal constitue une structure temporaire qui, de toute évidence, assure à la larve une fixation efficace. Excepté le cas particulier des femelles de Lernaeopodidae, chez lesquelles une partie du filament frontal est à l'origine du bouton fixateur, dans tous les autres cas connus, l'apparition de la phase adulte se traduit par une rupture du filament frontal et par un mode de fixation différent, d'une part de celui du copépodite et, d'autre part, de celui du Chalimus.

Cela nous amène à nous poser la question de savoir si l'utilisation d'antennes unciformes constitue un processus de fixation plus primitif que celui du filament frontal. Au niveau de l'efficacité de l'implantation, nous pensons qu'il n'en est rien, d'autant plus que chez Lernanthropus kroyeri ce mode de fixation se maintient chez l'adulte avec une pénétration de plus en plus accusée des crochets antennaires dans le filament branchial.

Pourtant, nous demeurons en accord avec Kabata (1981) pour considèrer que l'absence de filament frontal chez les larves de Copépodes Siphonostomes est un caractère primitif, en nous basant sur la comparaison avec le cycle évolutif des Copépodes Poecilostomes parasites de Poissons. Chez ces derniers, on peut distinguer deux types de cycles évolutifs : le premier où tous les stades Copépodites sont libres représenté par les Ergasilidae (Zmerzlaya, 1972 ; Ben Hassine et al., 1981), et le second où le Copépodite libre infestant se fixe par ses antennes et accomplit son développement sur l'hôte, représenté par les Chondracanthidae (Heegaard, 1947 ; Kabata, 1981). Il ne fait aucun doute que le cycle évolutif de Lernanthropus kroyeri montre des ressemblances étroites avec celui des Chondracanthidae appartenant à un ordre moins évolué.

Cela nous amène à considérer qu'au sein des Copépodes Siphonostomes parasites de Poissons, il est possible de se baser sur l'étude du développement post embryon- 
naire afin de distinguer deux lignées évolutives. L'une, primitive, proche des Poecilostomes les plus évolués (Chondracanthidae, par exemple) comprenant les Lernanthropidae, les Dichelesthiidae, les Dissonidae et, probablement, d'autres familles telles que les Hatschekiidae et les Pseudocycnidae caractérisée par des larves sans filament frontal ; l'autre, évoluée, comprenant les Caligidae, les Cecropidae, les Pennellidae, les Lernaeopodidae et bien d'autres familles, comme les Sphyriidae par exemple, caractérisée par la larve Chalimus. Ce qui nous conduit au schéma évolutif global suivant :

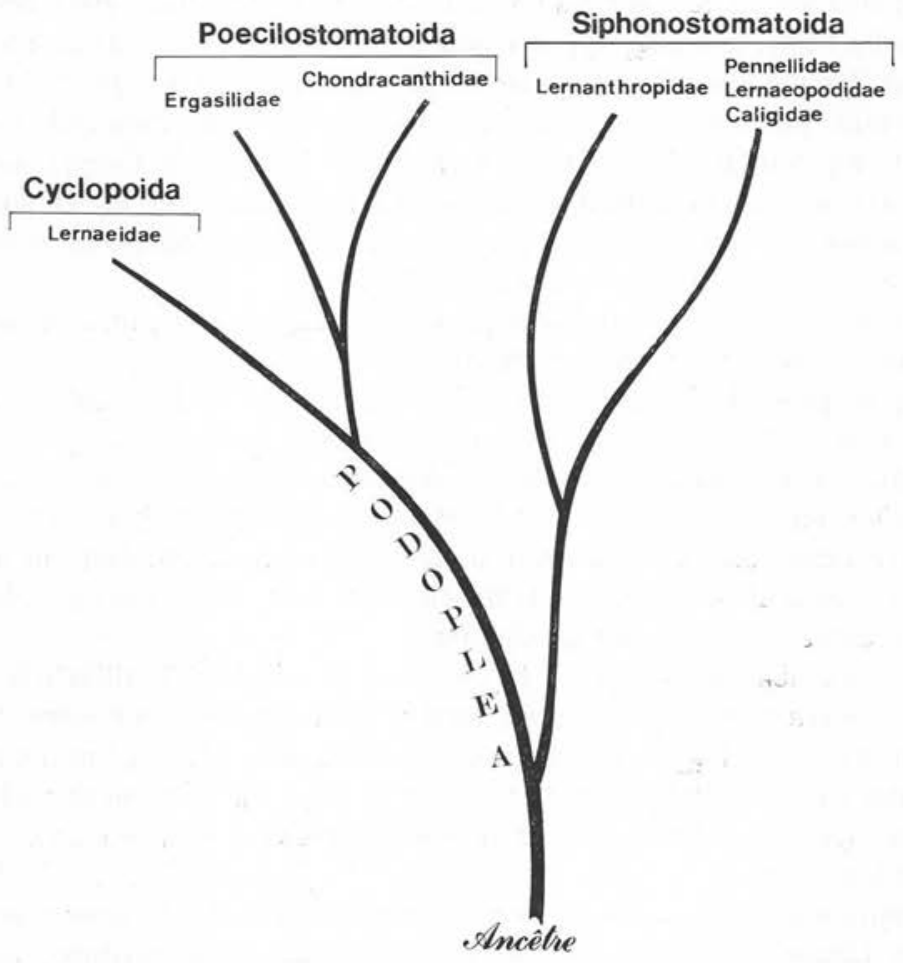

Arbre phylogénétique hypothétique des Copépodes parasites de Poissons établi d'après la morphologie (Kabata, I979) et les cycles évolutifs.

\section{BIBLIOGRAPHIE}

Anderson D. T., Rossiter G. T. : Hatching and larval development of Dissonus nudiventris Kabata (Copépoda, Fam. Dissonidae), a gill parasite of the Port Jackson Shark. Proc. Linn. Soc., N.S.W., 1969, 93, 476-481.

BARNABÉ G. : Contribution à la connaissance de la biologie du Loup Dicentrarchus labrax (L.) (Poisson Serranidae). Thèse U.S.T.L., 1976, 426 p.

Ben Hassine O. K., Rarbaut A. : Réalisation expérimentale du cycle évolutif d'Ergasilus lizae Kroyer, 1863 , Copépode parasite de Poissons Mugilidés. Premiers résultats de l’infestation. Arch. Inst. Pasteur Tunis, 1981, 58, 423-430. 
Bragoni G., Romestand B., Trilles J. P. : Parasitoses à Cymothoadien chez le Loup (Dicen-

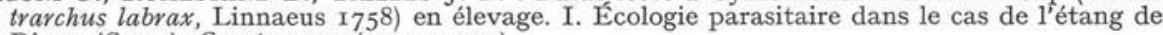
Diana (Corse). Crustaceana (sous presse).

Bragoni G., Romestand B., Trilles J. P.: Parasitoses à Cymothoadien chez le Loup (Dicentrarchus labrax, Linnaeus $175^{8}$ ) en élevage. II. Écophysiologie parasitaire dans le cas de l'étang de Diana (Corse). Ann. Parasitol. Hum. Comp. (sous presse).

Boxshall G. A. : The developmental stages of Lepeophtheirus pectoralis (Müller, I776) (Copepoda : Caligidae). J. Nat. Hist., r974, 8, 68I-700.

CABRal P. : Découverte d'un Caligide nouveau, Copépode parasite du tégument du Loup Dicentrarchus labrax (Linné, I 758). Rev. Trav. Inst. Pêch. Marit. (sous presse).

Grabda J. : Life-cycle and morphogenesis of Lernaea cyprinacea L. Acta Parasit. Pol., I963, 11, I69-198.

GRABDA J. : Contribution to knowledge of Cecrops latreillii Leach, I8I6 (Caligoida : Cercopidae) the parasite of the Ocean surfish Mola mola (L.). Acta Ichthyologica et piscatoria, 1973, 3, $6 \mathrm{I}-74$.

GURNEY $\mathrm{R}$. : The development of certain parasitic Copepoda of the Families Caligidae and Clavellidae. Proc. Zool. Soc., 1933-34, Is, 177-21 7.

HeEgaARd P. : Contribution to the Phylogeny of the Arthropods. Spolia Zool. Mus. Hauniensis, I947, 8, I-227.

Ho J. S. : Larval stages of Cardiodectes sp. (Caligoida : Lernaeoceriformes) a Copepod parasitic on fishes. Bull. Marc. Sci., 1966, I6, I 59-199.

Hwa T. K. : Studies on the life history of a fish-louse (Caligus orientalis) Cruser. Acta Zool. Sinica, I965, $17,48-57$.

IzAwA K. : Life-history of Caligus spinosus Yamaguti, r939 obtained from cultured yellow tail, Seriola quinquaradiata T. et S. (Crustacea : Caligoida). Rep. Fac. Fish. Prefect. Univ. Hie., 1969, 6, I27-157.

Kabata Z. : Developmental stages of Caligus clemensi Parker and Margolis, I964 (Copepoda : Caligidae). J. Fish Res. Board. Can., 1972, 29, I 57 I-1 593.

Kabata Z. : Parasitic Copepoda of British Fishes. The Ray Society, London, 1979, $45^{8} \mathrm{p}$.

Kabata Z. : Copepods (Crustacea) parasitic on Fishes : Problems and Perspectives. In : $A d v$. Parasitol., I981, 19, I-71.

Kabata Z., Cousens B. : Life cycle of Salmincola californiensis (Dana, 1852) (Copepoda : Lernaeopodidae). J. Fish. Res. Board. Can., I973, 30, 881-903.

Kabata Z., KHodorevsky O. A. : The Copepodid Stage of Dichelesthium oblongum (Abildgaard, 1794) a Copepod parasite of Acipenserid Fishes (en russe). Parazitologiya, 1977, 11, 236-240.

Kawatow K., Muroga K., Izawa K., Kasahara J. : Life-cycle of Allela macrotrachelus (Copepoda) parasitic on cultured black-sea-beam. J. Fac. Appl. Biol. Sci. Hiroshima Univ., r980,

19, I99-2I4.
LAMBERT A., Maillard C. : Parasitisme branchial simultané par deux espèces de Diplectanum Diesing $185^{2}$ (Monogenea, Monopisthocotylea) chez Dicentrarchus labrax (L. I758) (Téléostéen). C.R. Acad. Sci. Paris, 1974, 279, 1345-1347.

Lewis A. G. : Life history of the Caligid Copepod Lepeophtheirus dissimulatus, Wilson, 1905 (Crustacea : Caligoida). Pac. Sci., I963, 17 , I92-242.

Oliver G. : Effet pathogène de la fixation de Diplectanum aequans (Waganer, I857) Diesing, I858 (Monogenea Monopisthecotylea, Diplectanidae) sur les branchies de Dicentrarchus labrax (Linnaeus, 1758) (Pisces, Serranidae). J. Parazitenkd, 1977, 53, 7-1 I.

PAPERnA I. : Amyloodinium ocellatum (Brown, 193I) (Dinofiagellida) infestations in cultured marine fish at Eilat, Red Sea : Epizootiology and pathology. J. Fish. Dis., I980, 3, 363-372.

Paperna I., LAURENCIN F. B. : Parasitic infections of sea bass, Dicentrarchus labrax, and gilt head sea bream, Sparus aurata, in Mariculture facilities in France. Aquaculture, 1979, I6,

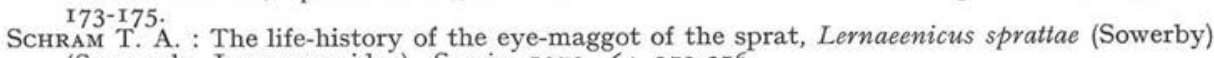
(Copepoda, Lernaeoceridae). Sarsia, 1979, 64, 279-316.

Scort Th., Scort A. : The British Parasitic Copepoda. The Ray Society, I9I3, Vol. I et 2, 252 p., $72 \mathrm{pl}$.

TESSEYRE C. : Étude des conditions d'élevage intensif du Loup Dicentrarchus labrax (L.). Thèse Doctorat $3^{e}$ Cycle, U.S.T.L. Monptellier, 1979, I 5 p.

Wilson C. B. : North American parasitic Copepoda belonging to the family Caligifae. Pt I-

Caliginae. Proc. U.S. Nat. Mus., I905, $28,479-672$.
Zmerzlaya E. I. : Ergasilus sieboldi Nordmann, I832, its development Biology and Epizootic significance. IZV, Gos. Nauchno-Issled. Inst. Ozern. Rechn. Rybn. Khoz., I972, 80, I32-I77. 